\title{
Effect of Mass Transfer and Hall Current on Unsteady MHD Flow with Thermal Diffusivity of a Viscoelastic Fluid in a Porous Medium
}

\author{
Anitha. $\mathrm{S}^{*}$, Lavanya. $\mathrm{M} * *$, Naganandhini. A***, Poornima. B**** \\ *(Assistant Professor,Department of Mathematics, PSGR Krishnammal college for women, Coimbatore-04 \\ Email: anithamaths21@gmail.com) \\ **(M.Sc Mathematics, PSGR Krishnammal college for women, Coimbatore-04) \\ ***(M.Sc Mathematics, PSGR Krishnammal college for women, Coimbatore-04) \\ ****(M.Sc Mathematics, PSGR Krishnammal college for women, Coimbatore-04)
}

\begin{abstract}
The paper investigated the effect of mass transfer and Hall current on unsteady MHD flow with Thermal Diffusivity of a viscoelastic fluid in a porous medium. The resultant equations have been solved analytically. The velocity, temperature and concentration distributions are derived, and their profiles for various physical parameters are shown through graphs. The coefficient of Skin friction, Nusselt number and Sherwood number at the plate are derived and their numerical values for various physical parameters are presented through tables. The influence of various parameters such as the thermal Grashof number, mass Grashof number, Schmidt number, Prandtl number, viscoelasticity parameter, Hartmann number, Hall parameter, and the frequency of oscillation on the flow field are discussed. It is seen that, the velocity increases with the increase in Gc, Gr, $\mathrm{m}$ and $\mathrm{K}$, and it decreases with increase in $\mathrm{Sc}, \mathrm{M}, \mathrm{n}$ and $\mathrm{Pr}$, temperature decreases with increase in $\operatorname{Pr}$ and $\mathrm{n}$, Also, the concentration decreases with the increase in $\mathrm{Sc}$ and $\mathrm{n}$.
\end{abstract}

Key Words:Viscoelastic, Hall Current, Magnetohydrodynamics, Porous medium.

\section{INTRODUCTION}

Magnetohydrodynamics (MHD) is a field of study which combines elements of electromagnetism and fluid mechanics to describe the flow of electrically conducting fluids. It is generally regarded as a difficult academic discipline, both conceptually as well as mechanically. MHD is the study of electrically conducting fluids, combining both principles of fluid dynamics and electromagnetism. Magnetohydrodynamics is the study of the magnetic properties of electrically conducting fluids. When a conducting fluid moves through a magnetic field, an electric field, may be induced and, in turn the current interacts with the magnetic field to produce a body force. The science which deals with this phenomenon is called Magnetohydrodynamics The subject of MHD is traditionally studied as a continuum theory, that is to say, attempts at studying discrete particles in the flows are not at a level such that computation in these regards is realistic. To run "realistic simulations" would require computations of flows with many more particles than current computers are able to handle. Aboeldahab and Elbardy (2001) examined Hall current effect on Magnetohydrodynamics free convection flow past a semi infinite vertical plate with mass transfer. Effect of Heat of Transfer on Unsteady Mhd Couette Flow Between Two Infinite Parallel Porous Plates In an Inclined Magnetic Field was investigated by Daniel Simon. Magaji investigated Unsteady MHD mixed convective oscillatory flow through a porous medium filled in a vertical channel with heat and mass transfer. Chemical Reaction and Hall Effect on MHD Convective Flow along an Infinite Vertical Porous Plate with Variable Suction and Heat Absorption was examined by Masthanrao, Balamurugan and Varma along with Raju. Ramana Murthy, Srinivasa Raju and Anand Rao studied The effects of Heat and Mass Transfer on MHD Natural Convective Flow Past an Infinite Vertical Porous Plate with Thermal Radiation and Hall Current.

Shit and Haldar investigated Combimed Effects of Thermal Radiation and Hall Current on MHD Free Convective Flow and Mass Transfer over a Stretching Sheet with Variable Viscosity. Thermo Diffusion and Chemical Reaction Effects on a Steady Mixed Convective Heat and Mass transfer Flow with Induced Magnetic Field was examined by Sravanthi, Leela Ratnam and Bhaskar Reddy. Hall Current Effects on Free Convective Flow of Stratified Fluid over an Infinite vertical Porous Plate was studied by Subbaiah Naidu. Radiation and mass 
transfer effects on unsteady MHD convective flow past an infinite vertical plate with Dufour and Soret effects was studied by Vedavathi, Ramakrishna and Jayarami Reddy.

\section{MATHEMATICAL FORMULATION}

We consider the unsteady flow of a viscous incompressible and electrically conducting viscoelastic fluid over an infinite porous plate with oscillating temperature and mass transfer. The $\mathrm{x}$ axis is assumed to be oriented vertically upwards along the plate and the y-axis is taken normal to the plane of the plate. It is assumed that the plate is electrically non - conducting and a uniform magnetic field of straight $B_{0}$ is applied normal to the plate. The induced magnetic field is assumed constant. So that $\vec{B}=(0, B, 0)$,. The plate is subjected to a constant suction velocity.

1. The equation of conservation of charge $\Delta \times J=0$, gives constants.

2. $\vec{J}=\omega_{e} \tau_{e}(\vec{J} \times \vec{E})=\sigma\left(\vec{V} \times \vec{B}+\frac{\Delta P_{e}}{e n_{e}}\right)$

Equation (1) reduces to

$$
\left.\begin{array}{l}
J_{x^{*}}=\frac{\sigma B_{0}}{\left(1+m^{2}\right)}\left(m u^{*}-\omega^{*}\right) \\
J_{y^{*}}=\frac{\sigma B_{0}}{\left(1+m^{2}\right)}\left(u^{*}-m \omega^{*}\right)
\end{array}\right\}
$$

Where

$\mathrm{m}=\omega_{e} \tau_{e}$

is the Hall parameter.

The governing equations for the momentum, energy and concentration are as follows;

$$
\begin{gathered}
\frac{\partial \mathrm{u}}{\partial \mathrm{t}}+v_{0} \frac{\partial u}{\partial y}=v \frac{\partial^{2} u}{\partial y^{2}}-k_{1} \frac{\partial^{3} u}{\partial y^{2} \partial t}-\sigma B_{0} \frac{(u+m \omega)}{\rho\left(1+m^{2}\right)} \\
+g \beta\left(T-T_{\infty}\right)+g \beta(C-C \infty)-\frac{v u}{k^{*}} \\
\frac{\partial \omega}{\partial \mathrm{t}}+v_{0} \frac{\partial \omega}{\partial y}=v \frac{\partial^{2} \omega}{\partial y^{2}}-k_{1} \frac{\partial^{3} \omega}{\partial y^{2} \partial t}-\sigma B_{0} \frac{(\omega+m u)}{\rho\left(1+m^{2}\right)} \\
-\frac{v \omega}{k^{*}}
\end{gathered}
$$

$$
\frac{\partial T}{\partial \mathrm{t}}+v_{0} \frac{\partial T}{\partial y}=\frac{K_{T}}{\rho C_{p}} \frac{\partial^{2} T}{\partial y^{2}}
$$

$$
\frac{\partial C}{\partial \mathrm{t}}+v_{0} \frac{\partial C}{\partial y}=D \frac{\partial^{2} C}{\partial y^{2}}+D_{1} \frac{\partial^{2} C}{\partial y^{2}}
$$

The boundary conditions of the problem are:

$$
\begin{aligned}
\mathrm{u}= & 0, \omega=0, \mathrm{~T}=\mathrm{T}_{\infty}+\left(\mathrm{T}_{\omega}-\mathrm{T}_{\infty}\right) \mathrm{e}^{\mathrm{int}}, \mathrm{C}=\mathrm{C}_{\infty} \\
& +\left(\mathrm{C}_{\omega}-\mathrm{C}_{\infty}\right) \mathrm{e}^{\mathrm{int}} \text { at } \mathrm{y}=0
\end{aligned}
$$

$\mathrm{u} \rightarrow 0, \omega \rightarrow 0, \mathrm{~T} \rightarrow 0, \mathrm{C} \rightarrow 0$ as $\mathrm{y} \rightarrow \infty$

Where $\mathrm{u}$ and $\mathrm{v}$ are the components of velocity in the $\mathrm{x}$ and $\mathrm{y}$ direction respectively, $\mathrm{g}$ is the acceleration due to gravity, $\beta$ and $\beta^{*}$ and are the coefficient of volume expansion, $\mathrm{K}$ is the kinematic viscoelasticity, $\rho$ is the density, $\mu$ is the viscosity, $\mathrm{V}$ is the kinematic viscosity, $\mathrm{K}_{\mathrm{T}}$ is the thermal conductivity, $C_{\mathrm{p}}$ is the specific heat in the fluid at constant pressure, $\sigma$ is the electrical conductivity of the fluid, $\mu_{\mathrm{e}}$ is the magnetic permeability, $\mathrm{D}$ is the molecular diffusivity, $D_{1}$ is the thermal diffusivity, $T_{\omega}$ is the temperature of the plane and $T_{\infty}$ is the temperature of the fluid far away from plane. $C_{\omega}$ is the concentration of the plane and $C_{\infty}$ is the concentration of the fluid far away from the plane.

And , $v=-v_{0}$, the negative sign indicate that the suction is towards the plane.

Introducing the following non-dimensionless parameters.

$$
\begin{aligned}
& \eta=\frac{\mathrm{v}_{0} \mathrm{y}}{\mathrm{v}}, \mathrm{t}=\frac{\mathrm{v}_{0} \mathrm{t}}{4 \mathrm{v}}, \mathrm{u}=\frac{\mathrm{u}}{\mathrm{v}_{0}}, \omega=\frac{\omega}{\mathrm{v}_{0}}, \\
& \theta=\frac{\mathrm{T}-\mathrm{T}_{\infty}}{\mathrm{T}_{\omega}-\mathrm{T}_{\infty}}, \mathrm{C}=\frac{\mathrm{C}-\mathrm{C}_{\infty}}{\mathrm{C}_{\omega}-\mathrm{C}_{\infty}}, \\
& \mathrm{Gr}=\frac{\mathrm{g} \beta \mathrm{v}\left(\mathrm{T}_{\omega}-\mathrm{T}_{\infty}\right)}{\mathrm{v}_{0}{ }^{2}}, \mathrm{Gc}=\frac{\mathrm{g} \beta \mathrm{v}\left(\mathrm{C}_{\omega}-\mathrm{C}_{\infty}\right)}{\mathrm{v}_{0}{ }^{2}}, \\
& \mathrm{M}=\frac{\sigma \mathrm{B}_{0}{ }^{2} \mathrm{v}}{\rho \mathrm{v}_{0}{ }^{2}}, \mathrm{Pr}=\frac{\mu \mathrm{C}_{\mathrm{p}}}{\mathrm{K}_{\mathrm{T}}}, \mathrm{Sc}=\frac{\mathrm{v}}{\mathrm{D}}, \mathrm{Sc}_{1}=\frac{\mathrm{v}}{\mathrm{D}}, \mid \\
& \mathrm{K}=\frac{\mathrm{k}_{1} \mathrm{v}_{0}{ }^{2}}{\mathrm{v}^{2}}, \mathrm{k}=\frac{\mathrm{k}^{*} \mathrm{v}_{0}{ }^{2}}{\mathrm{v}^{2}}
\end{aligned}
$$

Substituting the dimensionless variables in (8) into (3) to (6), we get (dropping the bars) 


$$
\begin{gathered}
\frac{1}{4} \frac{\partial \mathrm{u}}{\partial \mathrm{t}}-\frac{\partial u}{\partial \eta}=\frac{\partial^{2} u}{\partial \eta^{2}}-\frac{K}{4} \frac{\partial^{3} u}{\partial \eta^{2} \partial t}-M \frac{(u+m \omega)}{\left(1+m^{2}\right)} \\
-\frac{u}{k}+G r \theta+G c C \\
\frac{1}{4} \frac{\partial \omega}{\partial \mathrm{t}}-\frac{\partial \omega}{\partial \eta}=\frac{\partial^{2} \omega}{\partial \eta^{2}}-\frac{K}{4} \frac{\partial^{3} \omega}{\partial \eta^{2} \partial t}-M \frac{(\omega+m u)}{\left(1+m^{2}\right)} \\
-\frac{\omega}{k}
\end{gathered}
$$$$
\frac{1}{4} \frac{\partial \theta}{\partial \mathrm{t}}-\frac{\partial \theta}{\partial \eta}=\frac{1}{\operatorname{Pr}} \frac{\partial^{2} \theta}{\partial \eta^{2}}
$$$$
\frac{1}{4} \frac{\partial C}{\partial \mathrm{t}}-\frac{\partial C}{\partial \eta}=\frac{1}{S c} \frac{\partial^{2} C}{\partial \eta^{2}}+\frac{1}{S c} \frac{\partial^{2} C}{\partial \eta^{2}}
$$

The corresponding boundary conditions are $\mathrm{u}(0, \mathrm{t})=0, \omega(0, \mathrm{t})=0$,

$$
\begin{array}{r}
\theta(0, \mathrm{t})=\mathrm{e}^{\text {int }}, \mathrm{C}(0, \mathrm{t})=\mathrm{e}^{\text {int }} \text { at } \mathrm{y}=0 \\
\mathrm{u}(\infty, \mathrm{t})=\omega(\infty, \mathrm{t})=\theta(\infty, t)= \\
\mathrm{C}(\infty, \mathrm{t})=0 \text { as } \mathrm{y} \rightarrow \infty
\end{array}
$$

Equations (9) and (10) can be combined into a single equation by introducing the complex velocity.

$$
\mathrm{U}=\mathrm{u}(\eta, \mathrm{t})+\mathrm{i} \omega(\eta, \mathrm{t})
$$

Where

$$
\mathrm{i}=\sqrt{-1}
$$

Thus,

$$
\begin{gathered}
\frac{1}{4} \frac{\partial \mathrm{U}}{\partial \mathrm{t}}-\frac{\partial U}{\partial \eta}=\frac{\partial^{2} U}{\partial \eta^{2}}-\frac{K}{4} \frac{\partial^{3} U}{\partial \eta^{2} \partial t}- \\
M \frac{(1-i m)}{\left(1+m^{2}\right)}-\frac{U}{k}+G r \theta+G c C
\end{gathered}
$$

With boundary conditions:

$$
\begin{aligned}
\mathrm{U}(0, \mathrm{t})=0, \theta(0, \mathrm{t})=\mathrm{e}^{\mathrm{int}}, \mathrm{C}(0, \mathrm{t})=\mathrm{e}^{\mathrm{int}} \\
\text { at } \eta=0
\end{aligned}
$$

$\mathrm{U}(\infty, \mathrm{t})=\theta(\infty, t)=\mathrm{C}(\infty, \mathrm{t})=0$ as $\eta \rightarrow \infty$

where $\mathrm{Gr}$, is the thermal Grashof number, $\mathrm{Gc}$, is the mass Grashof number, $\mathrm{Sc}, \mathrm{Sc}_{1}$ is the Schmidt numbers, $\mathrm{Pr}$, is the Prandtl number, $\mathrm{K}$, is the viscoelastic Parameter, $M$, is the Hartmann number and $\mathrm{k}$, is the pearmeability.

\section{METHODS OF SOLUTION}

To solve (11), (12) and (15) subject to the boundary conditions (16), we assume solutions of the form

$$
\begin{aligned}
& \mathrm{U}(\eta, \mathrm{t})=U_{1}(\eta) e^{\mathrm{int}} \\
& \theta(\eta, \mathrm{t})=\theta_{1}(\eta) e^{\mathrm{int}}
\end{aligned}
$$

$C(\eta, \mathrm{t})=C_{1}(\eta) e^{\mathrm{int}}$

Where $U_{1}(\eta), \theta_{1}(\eta)$ and $C_{1}(\eta)$ are to be determined.

Substituting (17) to (19) into (11),(12) and (15), comparing harmonic and non harmonic terms, we obtain

$$
\begin{gathered}
\mathrm{U}^{\prime \prime}+\frac{U^{\prime}}{P_{1}}-P_{3} U=-\frac{G r \theta}{4 P_{1} e^{\mathrm{int}}}-\frac{G c C}{4 P_{1} e^{\mathrm{int}}} \\
\theta^{\prime \prime}+\operatorname{Pr} \theta_{1}^{\prime}-\frac{1}{4} \text { in } \operatorname{Pr} \theta_{1}=0 \\
\left(S c+S c_{1}\right) C^{\prime \prime}+S c S c_{1} C_{1}^{\prime}- \\
\frac{1}{4} i n S c S c{ }_{1} C_{1}=0
\end{gathered}
$$

And boundary conditions give

$$
\begin{array}{r}
\mathrm{U}_{1}(0)=, \theta_{1}(0)=\mathrm{C}_{1}(0)=1 \text { at } \eta=0 \\
\mathrm{U}_{1}(\infty) \rightarrow 0, \theta_{1}(\infty) \rightarrow 0, \mathrm{C}_{1}(\infty) \rightarrow 0 \\
\text { at } \eta=\infty
\end{array}
$$

where the primes represents differentiation with respect to . Solving (20) to (22) subject to the boundary conditions (23) and (25) and substituting the obtained solutions into (17) to (19) respectively.

Then the velocity field can be expressed as

$\mathrm{U}(\eta, \mathrm{t})=\left[\mathrm{A}_{6} \mathrm{e}^{-\mathrm{m}_{6} \eta}+\mathrm{A}_{7} \mathrm{e}^{-\mathrm{m}_{2} \eta}+\mathrm{A}_{8} \mathrm{e}^{-\mathrm{m}_{4} \eta}\right] \mathrm{e}^{\mathrm{int}}$ (24)

And the temperature field is given by

$\theta(\eta, \mathrm{t})=\mathrm{e}^{-\mathrm{m}_{2} \eta} \mathrm{e}^{\mathrm{int}}$

Similarly, the concentration distribution gives

$C(\eta, t)=e^{-m_{4} \eta} e^{i n t}$

\section{RESULTS AND DISCUSSION}

The effect of mass transfer and Hall current on unsteady MHD flow of a viscoelastic fluid in a porous medium has been formulated and solved analytically. In order to understand the flow of the fluid, computations are performed for different parameters such as Gr, Gc, Sc, Pr, n, M, K, and m.

\subsection{Velocity profiles}

Figures 1-8 represent the velocity profiles, figures 9 and 10 depict the temperature profiles and figures 11 and 12 show the concentration profiles with varying parameters respectively.

The effect of velocity for different values of $(\operatorname{Pr}=0.025,0.71,1,3)$ is presented in figure 1 , the graph show that velocity decreases with increase in Pr. The effect of velocity for different values of (Sc $=0.3,0.6,0.8,1,2.01)$ is given in figure 2 , the graph show that velocity decreases with the increase in Sc. Figure 3 denotes the effect of velocity for different values of $(\mathrm{n}=1,2,3,4,7)$, it is seen that velocity decreases with the increase in $n$. 
The effect of velocity for different values of $(\mathrm{m}=1$, $2,3,4,7$ ) is shown in figure 4 , it depict that velocity increases with increase in $\mathrm{m}$.

Figure 5 depicts the effect of velocity for $(M=1,2$, $3,4,7)$, the graph show that velocity decreases with the increase in $\mathrm{M}$.

The effect of velocity for different values of $(\mathrm{Gr}=1$, $2,3,4,7)$ is presented in figure 6 , it is seen that velocity increases with the increase in $\mathrm{Gr}$.

The effect of velocity for different values of $(\mathrm{Gc}=1$, $2,3,4,7$ ) is displayed in figure 7 , it is observed that velocity increases with the increase in Gc.

The effect of velocity for different values of $(\mathrm{K}=$ $0.0001,0.01,0.05,0.08,0.1)$ is shown in figure 8 , it is observed that velocity increases with the increase in $\mathrm{K}$.

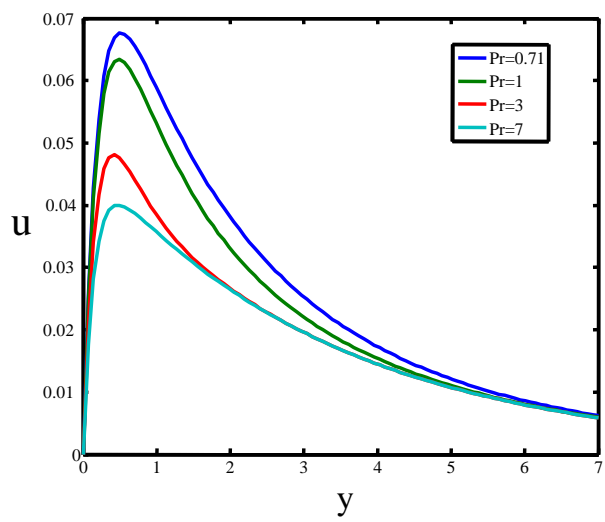

Figure 1: velocity profiles for different values of Pr.

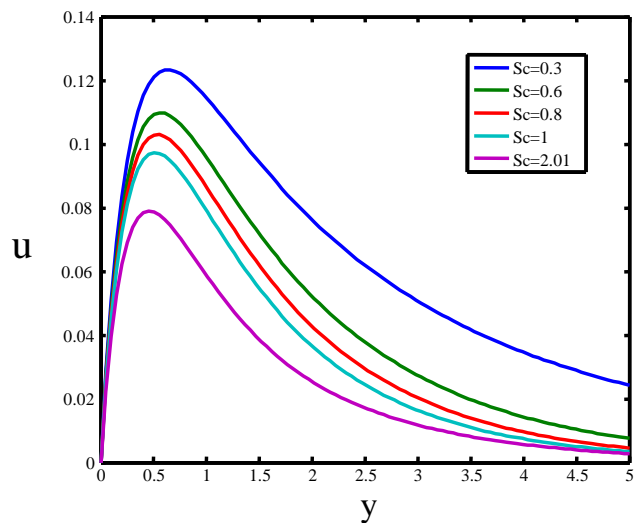

Figure 2: Velocity profiles for different values of Sc.

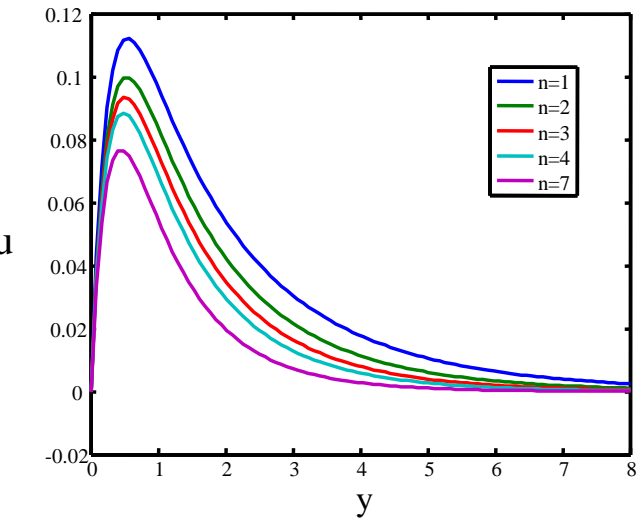

Figure 3: Velocity profiles for different values of $n$

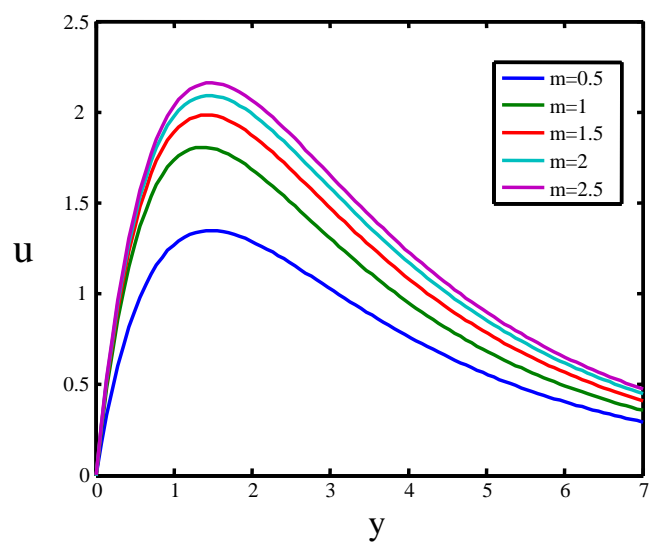

Figure 4: Velocity profiles for different values of $\mathrm{m}$.

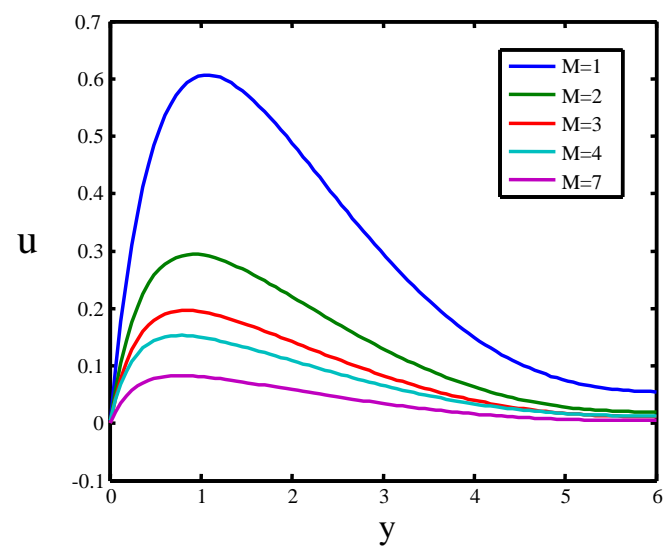

Figure 5:Velocity profiles for different $\mathrm{v}$ alues of $\mathrm{M}$. 


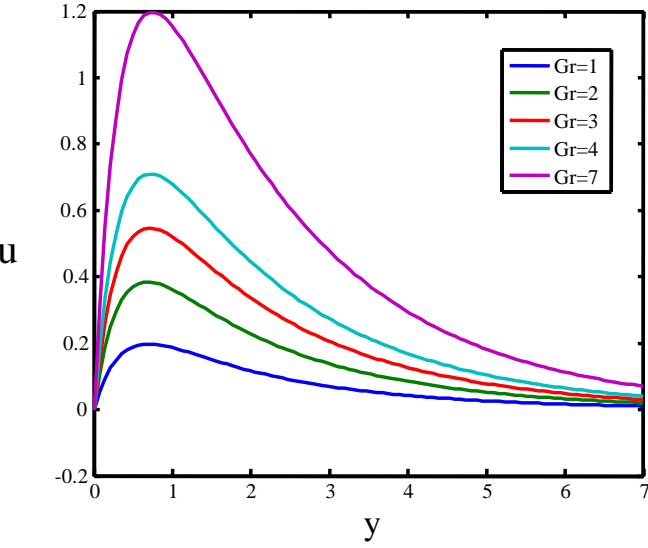

Figure 6: Velocity profiles for different values of $\mathrm{Gr}$.

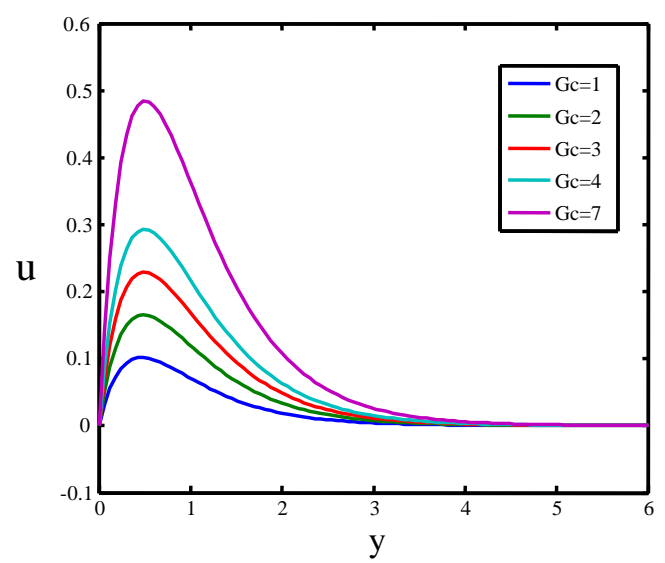

Figure 7: Velocity profiles for different values of Gc.

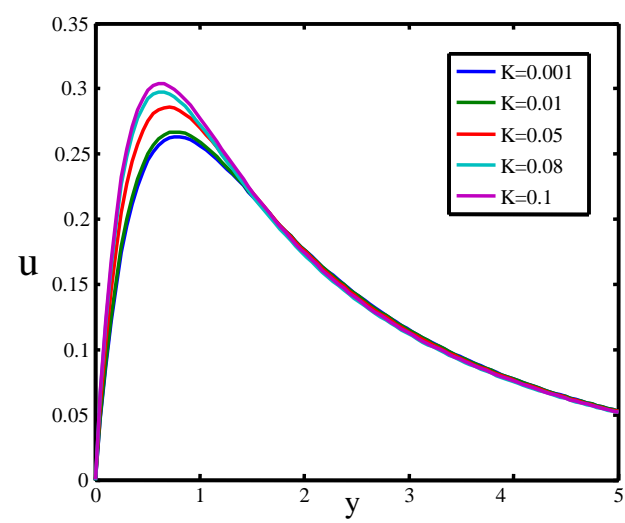

Figure 8: Velocity profiles for different values of K.

\subsection{Temperature profiles}

In figure 9 , the effect of temperature for different values of $(\operatorname{Pr}=0.025,0.71,1,3,7)$ is given. The graph show that temperature decreases with increasing Pr. Figure 10 shows the effect of temperature for different values of $(\mathrm{n}=0.025,0.71$, $1,3,7)$. The graph show that temperature decreases with decreasing $n$.

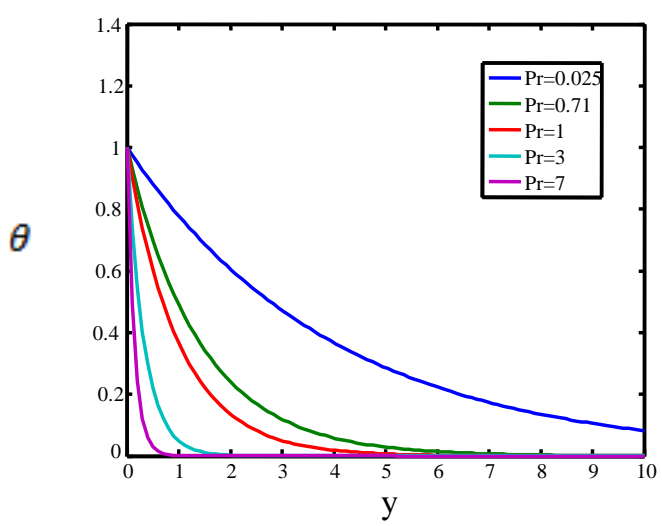

Figure 9: Temperature profiles for different values of $\mathrm{Pr}$.

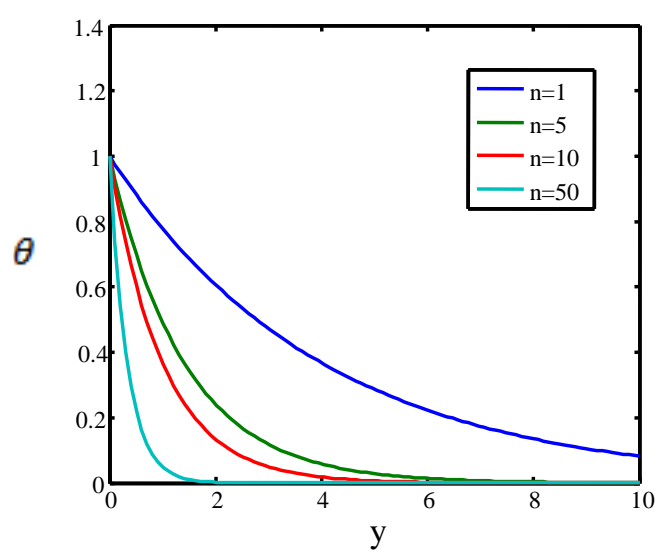

Figure 10: Velocity profiles for different values of $n$.

\subsection{Concentration profiles}

Figure 11 depicts the effect of concentration for $(\mathrm{Sc}=0.3,0.6,0.8,1,2.01)$, it is seen that concentration decreases with the increase in Sc. The effect of concentration for $(\mathrm{n}=0.3,0.6$, $0.8,1,2.01)$ is given in figure 12 , it is seen that concentration decreases with the decrease in $n$.

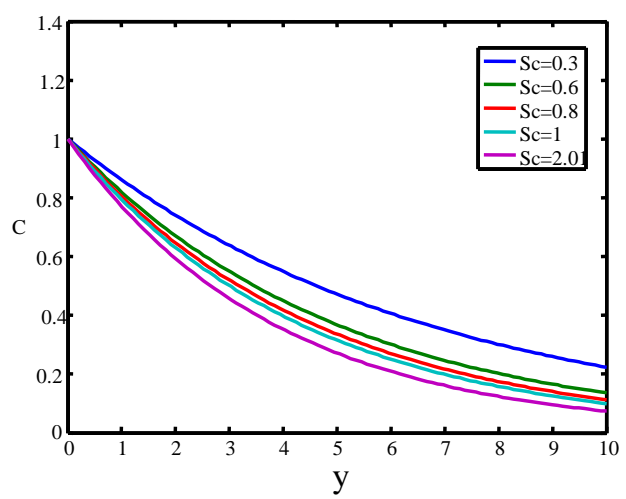

Figure 11: Concentration profiles for different values of Sc. 


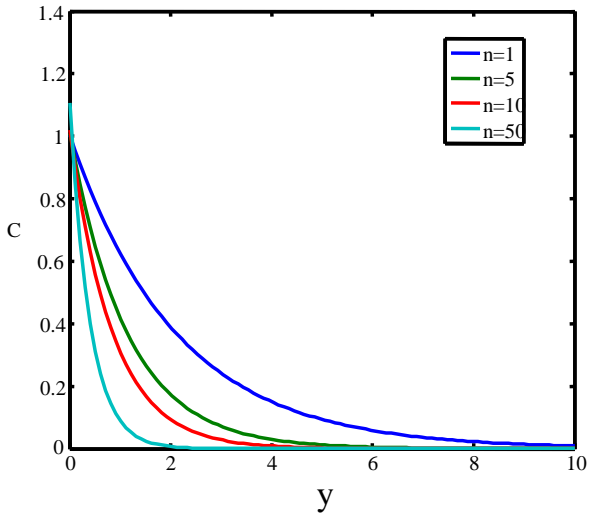

Figure

12: Concentration profiles for different values of $n$.

\section{SUMMARY AND CONCLUSION}

We have examined and solved analytically the governing equations for the effect of mass transfer and Hall current on unsteady MHD flow of a viscoelastic fluid in a porous medium analytically. In order to point out the effect of physical parameters namely; thermal Grashof number, mass Grashof number, Schmidt number, Prandtl number, viscoelasticity parameter, Hartmann number, Hall parameter and the frequency of oscillation on the flow field. We observe that, the velocity increases with the increase in $\mathrm{Gc}, \mathrm{Gr}, \mathrm{m}$, and $\mathrm{K}$, and it decreases with increase in Sc, $\mathrm{n}, \mathrm{M}$ and $\mathrm{Pr}$. Temperature decreases with increase in $\operatorname{Pr}$ and $n$, and concentration decreases with the increase in $\mathrm{Sc}$ and $n$.

\section{REFERENCES}

[1]. Aboeldahab, E. M. and Elbarby, E. M. E. (2001). Hall current effect on Magnetohydrodynamics free convection flow past a Semi - infinite vertical plate with mass transfer. Int. Journ. of Engineering Sciences. 39. $1641-1652$.

[2]. Daniel Simon (2014). Effect of Heat of Transfer on Unsteady Mhd Couette Flow Between Two Infinite Parallel Porous Plates In An Inclined Magnetic Field. International Journal of Mathematics and Statistics Invention (IJMSI). 2.2321-4759

[3]. Magaji AS. (2016).Unsteady MHD mixed convective oscillatory flow through a porous medium filled in a vertical channel with heat and mass transfer.Journal of Scientific and Engineering Research. 3(3). 2394-2630

[4]. Masthanrao. S, Balamurugan. K. S, Varma. S. V. K, and Raju.V. C. C. (2013). Chemical Reaction and Hall Effects on MHD Convective Flow along an Infinite Vertical Porous Plate with Variable Suction and Heat Absorption. Applications
AppliedMathematics: An International Journal(AAM). 8(1). 1932-9466.

[5]. Ramana Murthy. M. V, Srinivasa Raju. R, Anand Rao. J. ( 2015 ).Heat and Mass Transfer Effects on MHD Natural Convective Flow Past an Infinite Vertical Porous Plate with Thermal Radiation and Hall Current. Procedia Engineering. 127. $1330-1337$

[6]. Shit.G .S and Haldar. R. (2012). Combined Effects of Thermal Radiation and Hall Current on

[7]. MHD Free-Convective Flow and Mass Transfer over a Stretching Sheet with Variable Viscosity. Journal of Applied Fluid Mechanics. 5(2). 1735-3572

[8]. Sravanthi .C. S. Leela Ratnam. A. and Bhaskar Reddy. N. (2013). Thermo-Diffusion and Chemical Reaction Effects on a Steady Mixed Convective Heat and Mass Transfer Flow with Induced Magnetic Field. International Journal of Innovative Research in Science, Engineering and Technology. 2(9). 2319-8753

[9]. Subbaiah Naidu. K.Ch. V. (2016). Hall Current Effects on Free Convective Flow of Stratified Fluid over an Infinite vertical porous plate.IOSR Journal of Mathematics (IOSR-JM). 12(5). 2278-5728

[10]. Vedavathi. N, Ramakrishna. K, Jayarami Reddy. K. (2015). Radiation and mass transfer effects on unsteady MHD convective flow past an infinite vertical plate with Dufour and Soret effects. Ain Shams Engineering Journal. 6. 363-371

[11]. Takhar (2006). Unsteady flow free convective flow over an infinite vertical porous platedue to the combined effects of thermal and mass diffusion, magnetic field and Hall current. Journal of Heat and Mass Transfer. 39, 823 834.

$$
\begin{aligned}
& \text { APPENDIX } \\
& a=\sqrt{\frac{\operatorname{Pr}^{2}+i n \operatorname{Pr}}{4}}, m_{2}=\frac{\operatorname{Pr}}{2}+a, d=S c S c_{1} \\
& d_{1}=S c+S c_{1}, b=\sqrt{\frac{d^{2}+i n d d_{1}}{4 d_{1}^{2}}} \\
& m_{4}=\frac{d}{2 d_{1}+b, P_{1}=\left(1-\frac{i n K}{4}\right),} \\
& P_{2}=\left(\frac{i n}{4}+\frac{1}{k}+\frac{M(1-i m)}{\left(1+m^{2}\right)}, P_{3}=\frac{P_{2}}{P_{1}}\right. \\
& c=\sqrt{\frac{1+4 P_{1}^{2} P_{3}}{4 P_{1}^{2}}}, m_{6}=\frac{1}{2 P_{1}}+c,
\end{aligned}
$$




$$
\begin{aligned}
& A_{6}=-A_{7}-A_{8} A_{8}=-\frac{G c}{P_{1}\left(m_{4}{ }^{2}-\frac{m_{4}}{P_{1}}-P_{3}\right)} \\
& A_{7}=-\frac{G r}{P_{1}\left(m_{2}{ }^{2}-\frac{m_{2}}{P_{1}}-P_{3}\right)}
\end{aligned}
$$

